\title{
Feasibility and Field Performance of a Simultaneous Syphilis and HIV Point-of-Care Test Based Screening Strategy in at Risk Populations in Edmonton, Canada
}

\author{
Joshua Bergman, ${ }^{1}$ Jennifer Gratrix, ${ }^{1}$ Sabrina Plitt, ${ }^{2}$ Jayne Fenton, ${ }^{3}$ Chris Archibald, \\ Tom Wong, ${ }^{2}$ and Ameeta E. Singh ${ }^{1}$ \\ ${ }^{1}$ Alberta Health Services, Edmonton STI Clinic, 3B20 1111 Jasper Avenue, Edmonton, Alberta, Canada T5K 0L4 \\ ${ }^{2}$ Public Health Agency of Canada, 130 Colonnade Road, A.L. 6501H, Ottawa, ON, Canada K1A 0K9 \\ ${ }^{3}$ Provincial Laboratory for Public Health, 8440-112 Street, Edmonton, AB, Canada T6G 2J2 \\ Correspondence should be addressed to Ameeta E. Singh; ameeta@ualberta.ca
}

Received 5 June 2013; Accepted 13 November 2013

Academic Editor: Nitika Pant Pai

Copyright (C) 2013 Joshua Bergman et al. This is an open access article distributed under the Creative Commons Attribution License, which permits unrestricted use, distribution, and reproduction in any medium, provided the original work is properly cited.

Few studies have evaluated the feasibility of delivering syphilis point-of-care (POC) testing in outreach (nonclinical) settings in resource rich countries. The objectives of the study were to evaluate the feasibility and diagnostic performance of performing both HIV and syphilis POC testing in outreach settings and to document new cases identified in the study population. 1,265 outreach testing visits were offered syphilis and HIV POC testing and $81.5 \%(n=1,031)$ consented to testing. In our population, the SD Bioline 3.0 Syphilis Test had a sensitivity of $85.3 \%$ [CI (68.9-95.0)], specificity of $100.0 \%$ [CI (99.6-100.0)], positive predictive value (PPV) of $100.0 \%$ [CI (88.1-100.0)], and negative predictive value (NPV) of 99.5\% [CI (98.9-99.8)]. Test characteristics for the INSTI HIV-1/HIV-2 Antibody Test had a 100.0\% sensitivity [CI (39.8-100.00], 99.8 specificity [CI (99.3-100)], 66.7\% PPV [CI (22.3-95.7)], and 100.0\% NPV [CI (99.6-100.0)]. Four new cases of syphilis and four new HIV cases were diagnosed. In summary, at risk population seeking STI testing found POC tests to be acceptable, the POC tests performed well in outreach settings, and new cases of syphilis and HIV were identified and linked to treatment and care.

\section{Introduction}

In the late 1990s, Canada appeared to be on the verge of eliminating syphilis, as all but one province/territory had achieved rates of less than 0.5 per 100,000 population in 1997 [1]. In 2001, the reported rate of infectious syphilis started to increase rapidly, particularly among men, related to outbreaks occurring in large urban centres across Canada [2]. The majority of outbreaks across Canada have occurred among men who have sex with men (MSM) and individuals involved in sex trade, but other outbreaks have occurred among heterosexual persons not reporting risks associated with either of these populations [2,3]. Between 1999 and 2008, the province of Alberta experienced the largest increase in the reported rate of infectious syphilis in Canada and resulted in the province declaring a syphilis outbreak in March 2007 [2, 4]. The reported rate of infectious syphilis in
Edmonton, the second largest urban municipality in Alberta and with a population of over one million people for the census metropolitan area, was 8.1 per 100,000 in 2009, higher than the provincial rate of 7.4 per 100,000 [5]. The majority of cases in this ongoing outbreak were in heterosexual persons, but vulnerable populations such as MSM, people of aboriginal descent, sex workers, and people who inject drugs (IDU) have been disproportionately affected $[5,6]$. In 2011, $53 \%$ of reported male cases in the Edmonton zone occurred among MSM [7]. In 2010, the number of cases of infectious syphilis reported in Alberta and in Edmonton declined and continued to decrease to a reported rate of 3.2 per 100,000 in the Edmonton area in 2011 [8].

The return of infectious syphilis in Alberta has the potential to impact HIV control as individuals with syphilis have an estimated two-to-five fold increased risk of acquiring HIV [9]. Additionally, HIV positive individuals may be more 
infectious when coinfected with syphilis [10]. The reported rate of new HIV diagnoses was 7.9 per 100,000 population in Edmonton in 2011, with the majority of male cases reported among MSM, a group also affected by the resurgence of infectious syphilis in this area [11].

Standard syphilis and HIV testing in Alberta involves the collection and transportation of a specimen to one of two central laboratories, where it can take up to 10 days to receive reports on newly identified infections that require confirmatory testing. A retrospective review done at the Alberta Health Services (AHS) Edmonton STI Clinic in 1999 showed that approximately $17 \%$ of persons did not return for HIV test results [12]. Point-of-care (POC) tests have been of particular benefit in remote or resource-limited settings that may lack the infrastructure for laboratory-based screening tests, in populations that are traditionally more difficult to reach and where immediate results can influence patient care [13-17]. Even in resource rich countries, the ability to conduct the test in nontraditional settings, the rapid availability of results (usually in $<30$ minutes) and the elimination of loss to follow-up for test results may make this testing approach preferable to centralized laboratory screening in some settings [18].

A POC test has the potential to allow timely counselling, referral, and management and, in the case of syphilis, immediate treatment $[15-17,19]$. Although over a dozen commercially available syphilis POC tests are available in some regions of the world, there are currently no licensed syphilis POC tests in Canada [20, 21]. The SD Bioline 3.0 is a syphilis POC test that has shown comparable performance to standard tests when used in prenatal or high risk population in low income countries and at the point-of-care [22]. Presently, one HIV POC test is licensed for use at the pointof-care in Canada, the INSTI HIV-1/HIV-2 Antibody Test Kit. Although the kit has been evaluated in acute care settings in Alberta, where the rapid test is being performed in a laboratory setting, it has not been evaluated at the POC in nonclinical, community-based settings serving hard to reach population [23]. POC testing for syphilis has never been offered and evaluated in Alberta.

The primary objective of this project was to evaluate the feasibility of performing both HIV and syphilis POC testing in outreach settings in populations affected by the ongoing syphilis outbreak. HIV POC testing was included in the study as HIV status impacts the recommended treatment for infectious syphilis [24]. The secondary objective was to evaluate the diagnostic performance of rapid POC tests against standard laboratory tests. The tertiary objective was to document new cases of syphilis and HIV identified in the study population and their linkage to treatment and care. Our hypotheses were that POC testing would be acceptable to Edmonton's at risk population and that test results would be comparable to standard testing.

\section{Materials and Methods}

2.1. Study Location and Population. Between February 14, 2011 and August 28, 2012, the Edmonton STI Clinic outreach team (comprised of Registered Nurses (RN) and Outreach support workers) offered POC testing to individuals 18 years of age and older who consented to standard STI testing. Both syphilis and HIV POC testing were conducted on whole blood obtained from finger prick specimens. Individuals could participate more than once. Individuals who self reported they were HIV positive did not undergo HIV POC testing, but individuals self reporting a previous syphilis diagnosis were not excluded from syphilis POC testing. POC tests were offered to individuals at outreach locations including two correctional facilities, three inpatient addictions facilities (one site is a male only residential program), one health centre, several community-based organizations such as inner city drop-in centres, organizations serving sex trade workers, and four sites visited by MSM (e.g., bathhouse and gay bars). These sites were selected as they were existing outreach sites where STI testing was already offered on a routine basis and did not require additional logistical or human resource support; however, they also provided access to the key populations that have been represented in the Alberta outbreak to date, such as Aboriginal people, people who inject drugs, those involved in the sex trade, and MSM who have made up the majority of reported male cases since 2010 in Edmonton.

Based on previous testing conducted by the STI Outreach team, we estimated that the prevalence of reactive syphilis tests among our population would be $6.3 \%$. At $6.3 \%$ reactivity and with 1,000 individuals enrolled, we estimated that we would be able to identify a POC test sensitivity of $87.5 \%$ $( \pm 8 \%)$.

2.2. Testing. POC test results were compared to standard laboratory screening tests performed at the Alberta Provincial Laboratory for Public Health (ProvLab) on serum samples collected simultaneously from participants.

2.2.1. Syphilis. The POC syphilis test used was the SD Bioline Syphilis 3.0 (SD Bioline 3.0 Test, Standard Diagnostics, Inc., Korea), which is a solid phase immunochromatographic assay for the qualitative detection of antibodies of all isotypes (IgG, IgM, and IgA) against Treponema pallidum (TP), with an expected result within 5-20 minutes [25]. The standard laboratory test used for syphilis was a treponemalspecific enzyme immunoassay (EIA) (Architect Syphilis TP Microparticles, Abbott Laboratories, Illinois, USA), which was the initial screen [26]. Quantitative RPR titre was obtained on all samples tested reactive by the syphilis EIA (or Syphilis POC test) [26]. A line immunoassay (INNOLIA Syphilis, Innogenetics NV, Ghent, Belgium) was used as the confirmatory test for samples submitted from individuals with no history of confirmed syphilis serology [26]. The EIA was used as the reference test for individuals who had a negative POC test (with no previous history of syphilis diagnosis and treatment) and for those with a positive POC test with a previous history of syphilis diagnosis and previously confirmed positive with the INNO-LIA. The INNO-LIA was used as the reference test for individuals with a positive POC test and no previous history of syphilis diagnosis and not previously confirmed positive with the INNO-LIA. 
2.2.2. HIV. The HIV POC test used was the INSTI HIV1/HIV-2 Antibody test (INSTI HIV-1/HIV-2 Antibody Test, bioLytical Laboratories, Richmond, Canada). This is a manual, visually read, flow-through immunoassay for the qualitative detection of antibodies to HIV Type 1 and Type 2 in human EDTA-whole blood, fingerstick blood, serum, or EDTA-plasma in as little as 60 seconds [27]. For HIV, a third generation EIA (AxSym HIV 1/2 gO, Abbott Laboratories, Illinois, USA) was the initial standard laboratory screening test [26]. On June 14, 2011, ProvLab began using a fourth generation EIA (Architect HIV Ag/Ab Combo, Abbott Laboratories, Illinois, USA) as the initial screening test [26, 28]. Samples tested reactive by standard EIA screen or by HIV POC testing from individuals with no history of confirmed HIV serology were tested by a second EIA (Biorad Genetic Systems HIV-1/HIV-2 PLUS O EIA, BioRad Laboratories, California, USA) and HIV Western Blot (Genetic Systems HIV-1 Western Blot, BioRad Laboratories, California, USA) [26].

2.2.3. Quality Control. In order to ensure the integrity of the testing materials, a control and proficiency testing program was established by the Laboratory Study Coordinator. Upon receipt of a new lot number of rapid kits, a lot release panel was performed and evaluated prior to the release of kits to storage. Kits were stored in the laboratory where temperatures were monitored on a daily basis. Additionally, the Laboratory Study Coordinator would run positive and negative controls on each new box of kits prior to distribution to the Outreach Team. The Provincial Laboratory also participated in a College of American Pathologists survey three times a year, thereby validating the proficiency of the supporting laboratory.

2.3. Patient Management. Algorithms for both syphilis and HIV POC testing were developed to guide testing, treatment, partner notification, and reporting. Syphilis treatment and counselling were based on the POC test result, previous syphilis testing and treatment, presence of signs and symptoms suggestive of syphilis, and the participant's sexual history. For those testing positive for syphilis by POC testing, the study nurse would contact staff at the STI Clinic to access the provincial STI database for a prior history of syphilis diagnosis and treatment in Alberta. All positive syphilis tests without a prior history of treatment were reviewed by phone with a STI consultant (AES) and a decision was made to provide immediate treatment or not. The POC syphilis test result was just one factor in the clinical decision algorithm for immediate treatment. Given the relative safety of treatment (benzathine penicillin G-long acting [BicillinLA]) and the potential harm posed by untreated syphilis, it is expected that the benefits of potential overtreatment outweigh any potential harm, especially in the context of hard-to-reach population who are more likely to be lost to follow-up. For those testing positive for HIV through POC, the result and case were reviewed by phone with a STI consultant (AES) to determine whether any immediate referral or management was required. Patients were informed that the syphilis or HIV POC test result was "preliminary" and the parallel standard testing being performed would confirm the result. All individuals with positive standard test results were contacted and actively followed up for necessary treatment and referral. For HIV positive results, this included referral to an HIV clinic.

2.4. Data Collection and Analyses. Standardized data collection forms were used by the nursing staff to collect and record demographics, clinical, and risk information on clients through a verbal history. All data was then entered into a password protected Microsoft Access database.

Feasibility was defined as the proportion of individuals consenting to POC divided by the total number of participants offered POC testing. To determine differences between participants consenting and those declining POC testing, age, gender, and testing location were compared using chi-square for categorical variables and Mann-Whitney $U$ test for age. A two sided $P$ value of $<0.05$ was considered statistically significant. A descriptive analysis of those individuals who had both syphilis and HIV POC testing was completed and due to the gender based differences in sex and drug behaviour, gender stratified analyses were also conducted to better understand our patient population. These analyses were performed using IBM SPSS Statistics 19 (IBM, Armonk, New York, USA). Diagnostic performances of POC test results were compared to standard testing results and test performance characteristics and $95 \%$ binomial confidence intervals were calculated (Stata version 11.0, StataCorp LP, College Station, Texas, USA). Cases were defined as having a new infection if the provincial databases for HIV and STI had no previous record of infection.

2.5. Ethics/Approvals. Ethics are obtained from the University of Alberta and Health Canada/Public Health Agency of Canada Research Ethics Boards. Special Access Approval was granted from Health Canada's Medical Devices Special Access Programme for the SD Bioline Syphilis 3.0 Test. Approval was obtained to provide POC treatment for syphilis based on a combination of the SD Bioline Syphilis 3.0 Test result as well as clinical information available at the time of testing (see Section 2.3).

\section{Results}

3.1. Feasibility of POC Testing. A total of 1,183 individuals at 1,265 outreach testing visits were offered syphilis and HIV POC testing and $81.5 \%(n=1,031)$ consented to testing. The majority of visits took place in locations where clients resided at the time of testing, correctional facilities $(50.2 \%$; $n=635)$ and inpatient addictions facilities $(26.8 \% ; n=339)$. Ten percent $(n=126)$ of testing visits took place in locations serving MSM (Table 1).

There was no difference in gender or median age among visits where the participant consented to POC testing compared to those visits where POC testing was declined. Acceptance varied between testing sites with the highest acceptance among testing sites for MSM and the lowest acceptance at community-based organizations. 
TABLE 1: Characteristics of participants offered syphilis and/or HIV POC testing $(N=1,265)$.

\begin{tabular}{lccc}
\hline & Accepted $(n=1031)$ & Declined $(n=234)$ & Total $(n=1265)$ \\
\hline Sex & & & \\
$\quad$ Female & $272(26.4)$ & $71(30.3)$ & $343(27.1)$ \\
$\quad$ Male & $759(73.6)$ & $163(69.7)$ & $922(72.9)$ \\
\hline Median age (IQR) & $30(25-39)$ & $29(24-38)$ & $30(24-39)$ \\
\hline Testing site & & & \\
$\quad$ Corrections & $508(80.0)$ & $127(20.0)$ & 635 \\
Inpatient addictions & $291(85.8)$ & $48(14.2)$ & 339 \\
Health facility & $39(73.6)$ & $14(26.4)$ & 53 \\
Community-based organization & $78(69.6)$ & $34(30.4)$ & 112 \\
MSM & $115(91.3)$ & $11(8.7)$ & 126 \\
\hline
\end{tabular}

IQR: interquartile range.

${ }^{a}$ Compares participants who accepted and those who declined using chi square and Mann Whitney tests.

3.2. Characteristics of Study Population. In 1,031 visits, participants consented to either syphilis and/or HIV POC testing: in 1,024 visits, syphilis POC testing was performed (2 visits declined syphilis POC testing and 5 visits syphilis POC testing kits were not available) and in 1,012 visits, HIV POC testing was performed (11 participants declined HIV POC testing; 5 were previously positive for HIV and therefore did not meet inclusion criteria, and in 3 visits, HIV POC testing kits were not available). In 1,004 visits, both syphilis and HIV POC testing was performed on 951 individuals (Table 2). The majority of participants were male $(73.3 \%)$, reported a history of substance use (non-IDU: $81.5 \%$ and IDU: $25.6 \%$ ), shared of drug equipment (58.5\%), and had previously been tested for HIV (82.6\%). Nearly one-half of female participants $(44.5 \%)$ reported a history of sex trade and nearly onequarter $(22.1 \%)$ of males reported sex with a sex trade worker. Nearly one-fifth $(18.9 \%)$ of male participants reported the same sex partnering. Gender-stratified analysis indicates that in comparison to males, female participants were more likely to be younger (median 29 years versus 30 years $P=0.04$ ), Gender-stratified analysis indicates that in comparison to males, female participants were more likely to be younger (median 29 years versus 30 years $P=0.04$ ), be of Aboriginal descent $(57.7 \%$ versus $39.0 \%, P<0.001)$, report a history of IDU (35.4\% versus $22.0 \%, P<0.001$ ), and to be previously syphilis seropositive (7.1\% versus $1.9 \%, P<0.001)$.

3.3. Diagnostic Performance of POC Tests. Five syphilis POC tests $(0.49 \%)$ were false negatives when compared to the standard laboratory test; four were in individuals previously treated for syphilis and one was newly diagnosed, staged, and treated for late latent syphilis. There were no false positive syphilis POC test results.

Compared with standard serological testing, the SD Bioline 3.0 Syphilis Test had a sensitivity of $85.3 \%$ [CI (68.9-95.0)], specificity of $100.0 \%$ [CI (99.6-100.0)], positive predictive value (PPV) of $100.0 \%$ [CI (88.1-100.0)], and negative predictive value (NPV) of 99.5\% [CI (98.9-99.8)] (Table 3).

Compared with standard serological testing, the INSTI HIV-1/HIV-2 Antibody Test had a $100.0 \%$ sensitivity [CI (39.8-100.00], 99.8 specificity [CI (99.3-100)], 66.7\% PPV
TABLE 2: Demographic and risk behaviour characteristics of individuals who received syphilis and HIV POC testing $(N=951)$.

\begin{tabular}{|c|c|c|}
\hline Characteristic & $n$ & $\%$ \\
\hline Male gender & 697 & 73.3 \\
\hline Median age (years, IQR) & 30 & $25-39$ \\
\hline \multicolumn{3}{|l|}{ Ethnicity } \\
\hline Aboriginal $^{\mathrm{a}}$ & 406 & 44.0 \\
\hline Caucasian & 449 & 48.6 \\
\hline Other ethnicities & 68 & 7.4 \\
\hline No permanent address & 185 & 24.2 \\
\hline \multicolumn{3}{|l|}{ Sexual partnering } \\
\hline Heterosexual exclusively & 753 & 79.9 \\
\hline Same sex ${ }^{\mathrm{b}}$ & 189 & 20.1 \\
\hline \multicolumn{3}{|l|}{ Sexual behaviour } \\
\hline Median age of sexual debut (IQR) & 14 & $13-16$ \\
\hline \# sexual partners in last 6 months & 2 & $1-3.5$ \\
\hline History of sex work (females only) & 113 & 44.5 \\
\hline History of sex work (males only) & 6 & 0.9 \\
\hline Sex with a sex worker (males only) ${ }^{c}$ & 154 & 22.1 \\
\hline \multicolumn{3}{|l|}{ Substance use } \\
\hline History of non-IDU & 776 & 81.6 \\
\hline History of IDU & 243 & 25.6 \\
\hline History of sharing drug equipment & 556 & 58.5 \\
\hline \multicolumn{3}{|l|}{ Laboratory test results } \\
\hline Previous HIV test & 725 & 82.6 \\
\hline HIV seropositive & 4 & 0.4 \\
\hline Syphilis seropositive & 31 & 3.3 \\
\hline
\end{tabular}

IQR: interquartile range.

IDU: injection drug use.

${ }^{a}$ Includes First Nations, Inuit, and Métis.

${ }^{\mathrm{b}}$ Includes individuals who reported sex with both males and females.

${ }^{\mathrm{c}}$ No female participants reported sex with a sex worker.

[CI (22.3-95.7)], and 100.0\% NPV [CI (99.6-100)], when compared to standard serological testing (Table 3 ).

3.4. New Cases and Linkages to Care. Of 1,024 syphilis POC tests, $29(2.8 \%)$ were positive, and of these, 25 (86.2\%) were in individuals previously treated for syphilis with no evidence 
TABle 3: Performance characteristics of syphilis and HIV point-of-care testing.

\begin{tabular}{|c|c|c|c|c|}
\hline Factor & Positive (\%) & Negative (\%) & Total (\%) & Performance $^{\mathrm{a}}(\% ; 95 \% \mathrm{CI})$ \\
\hline \multicolumn{5}{|l|}{ Syphilis ${ }^{b}$} \\
\hline POCT result & $29(2.8)$ & $990^{\mathrm{c}}(97.2)$ & $1019(100.0)$ & \\
\hline Standard test Result & $34(3.3)$ & $985(96.7)$ & $1019(100.0)$ & \\
\hline Sensitivity & & & & $85.3(68.9-95.0)$ \\
\hline Specificity & & & & $100.0\left(99.6-100^{\mathrm{d}}\right)$ \\
\hline PPV & & & & $100.0\left(88.1-100^{\mathrm{d}}\right)$ \\
\hline NPV & & & & $99.5(98.8-99.8)$ \\
\hline \multicolumn{5}{|l|}{$\mathrm{HIV}^{\mathrm{e}}$} \\
\hline POCT result & $6(0.6)$ & $993^{f}(99.4)$ & $999(100.0)$ & \\
\hline Standard test Result & $4(0.5)$ & 995 (99.6) & $999(100.0)$ & \\
\hline Sensitivity & & & & $100.0\left(39.8-100^{\mathrm{d}}\right)$ \\
\hline Specificity & & & & $99.8(99.3-100)$ \\
\hline PPV & & & & 66.7 (22.3-95.7) \\
\hline NPV & & & & $100.0\left(99.6-100^{\mathrm{d}}\right)$ \\
\hline \multicolumn{5}{|c|}{ 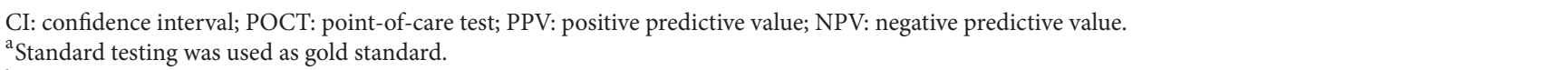 } \\
\hline \multicolumn{5}{|c|}{$\begin{array}{l}{ }^{b} \text { Calculations do not include } 1 \text { invalid POCT result (negative on follow-up standard testing). } \\
\text { c Does not include } 4 \text { negative specimens which did not have standard follow-up testing performe } \\
\text { unable to draw blood }(n=1) \text { ). } \\
\text { d One-sided, } 97.5 \% \text { confidence interval. } \\
{ }^{e} \text { Calculations do not include } 3 \text { invalid POCT results (negative on follow-up standard testing). }\end{array}$} \\
\hline
\end{tabular}

of a new syphilis infection (Table 3 ). The remaining four positive tests were in individuals with no previous history of syphilis diagnosis and treatment. Two of the individuals, one tested in a community-based setting and the other incarcerated, were treated presumptively at the time of testing and were subsequently staged and treated as early latent and late latent syphilis, respectively. The other two individuals, who were incarcerated and not considered at risk of being lost to follow-up nor at risk of transmitting syphilis before treatment, had their treatment delayed (5 and 19 days, resp.) to ensure adequate staging and treatment. These cases were subsequently staged as early latent and late latent syphilis and were treated while incarcerated.

Of 1,012 HIV POC tests, 6 (0.6\%) were reactive; four were newly diagnosed HIV cases as confirmed by Western Blot and two were false positive test results, confirmed HIV negative by the BioRad EIA and Western Blot (Table 3). All four individuals were informed of their positive standard HIV test result within two weeks and were referred to an infectious disease specialist for HIV care.

\section{Discussion}

Our study is the first pilot of dual syphilis and HIV POC testing in outreach settings among at risk populations in North America.

4.1. Feasibility of POC Testing. Results from our study suggest that POC tests for syphilis and HIV were well accepted among at risk population in outreach settings in Edmonton. A significant proportion of individuals who consented to syphilis and HIV POC testing were incarcerated and reported substance use and sex work. The proportion of risk behaviours among our participants was similar to that individuals diagnosed with infectious syphilis in Edmonton during the outbreak [6].

The settings that had the highest acceptance of testing were those accessed by MSM such as a bathhouse, with 91.3\% consenting to POC testing. The high acceptance of POC testing at MSM sites is supported by American research which showed significantly higher acceptance of rapid HIV testing than standard testing when offered in a bathhouse setting [29]. Almost half of the participants in this study were incarcerated and this group had a high acceptability of POC testing, as $80.0 \%$ consented to POC syphilis testing. This compares well to a US study, which showed $88 \%$ of inmates consenting to rapid HIV testing in a Rhode Island Department of Corrections jail [30]. The second most common setting where POCT was offered in our study was inpatient addiction treatment centres, where $85.8 \%$ of individuals offered POCT consented. This is higher than that of two US studies, which found $62 \%$ and $74 \%$ of individuals, respectively, consenting to rapid HIV testing in two separate inpatient substance abuse treatment programs [31, 32]. The higher rate of acceptance in this study could be related to POC testing only being offered to individuals who had already requested standard STI testing in outreach locations while the two US studies offered rapid testing to all individuals within their respective programs. 
Although the reasons for declining POC tests were not examined, we found that our lowest acceptance rate was in community-based organizations. Numerous studies in North America have cited reasons for declining rapid HIV testing, particularly among high risk population in outreach settings, including the extra time taken to conduct the POC test, not being prepared to receive a HIV result the same day as the test, recent testing, the perception that they were not at risk for HIV infection, and the venue for testing [30-33]. Future research should evaluate the acceptability of syphilis POC testing, among at risk populations in outreach settings.

4.2. Diagnostic Performance of POC Tests. Multiple studies in the USA and Mexico have evaluated the technical performance of various rapid syphilis testing platforms [34-37]. The performance of the syphilis and HIV POC tests used in this study is similar to that reported in other studies $[22,23,38]$. A prospective multicentre clinic-based evaluation of the SD Bioline 3.0 Syphilis Test showed sensitivity ranging from 85.7 to $100 \%$ and specificity ranging from 98.1 to $99.4 \%$, using whole blood in a clinic based setting, across four different sites [22]. A recent systematic review and meta-analysis of treponemal rapid POC tests for syphilis reported a sensitivity of $84.50 \%(78.81,92.61)$ and specificity of $97.95 \%(92.54,99.33)$ for the SD Bioline 3.0 Syphilis Test [38]. This compares to a lower sensitivity range of $85.3 \%$ (68.9-95.0) reported in this study. This might not be related to the test itself but rather to the environment or operator of the kit, for example, suboptimal sample volume being applied to the kit [19]. The lower sensitivity range reported in this study for the syphilis POC test would be a potential risk of missed opportunity for treatment. The field performance of the INSTI HIV-1/HIV-2 in this study was comparable to the report of a pilot study in Alberta, when the test was carried out in a controlled laboratory setting [23].

4.3. New Cases and Linkages to Care. We had hoped that syphilis POC testing would identify new infectious syphilis cases and allow POC treatment in affected populations in our region and thus assist with outbreak control. However the majority of individuals testing positive using the syphilis POC test in this study had been previously diagnosed and treated for syphilis (25 of 29 positive tests) and only four newly diagnosed cases of syphilis were identified (two cases of early latent and two cases of late latent syphilis). Half of the newly diagnosed cases received immediate treatment and in the other half, treatment was deferred but completed at a later date, as these participants were not at a risk of being lost to follow-up due to incarceration and not having an imminent release date. These data highlight the limited usefulness of treponemal POC tests in providing POC treatment to a previously syphilis seropositive population as the test is unable to distinguish between new and old infections. Unnecessary treatment may be provided unless quantitative nontreponemal testing is available when testing previously treated cases [39]. None of the 25 previously diagnosed syphilis cases identified in this study by POC testing were retreated because the testing $\mathrm{RN}$ was able to access the provincial STI database at the time of care to verify previous diagnosis and treatment. However in many field settings, the ability to verify previous diagnosis and treatment may not be possible. We postulate that POC testing in this outreach setting would have identified more cases in the earlier stages of the outbreak which peaked in 2006 at 10.9 per 100,000 but had declined to 3.4 per 100,000 and 3.2 per 100,000 in 2011 and 2012, respectively [8]. Dual treponemal and nontreponemal tests have the potential to address the issue of distinguishing previous infections from new infections. A recent study in China reported good test performance of a dual syphilis POC test when used in STI Clinic and outreach settings [40]. Future research is needed to evaluate the impact of using the dual syphilis POC test on uptake and intervention among high risk groups. The treponemal SD Bioline Syphilis 3.0 test was chosen for this study as no reliable dual syphilis POC test was available at the time the study was initiated.

The World Health Organization recommends assessing the existing quality of and access to syphilis testing and treatment for a population in determining the utility of introducing syphilis POC testing [41]. Standard syphilis testing is widely available in Alberta and is performed in accredited laboratories by skilled staff. However, the Alberta syphilis outbreak highlighted that those at highest risk do not necessarily access traditional testing settings, as evidenced by the increase in congenital syphilis cases, many of whom were born to street-involved women who had not accessed prenatal care which includes screening for syphilis [42]. The Outreach Team performing this study was formed in response to this observation and it was hoped that this population would be reached more readily and provided access to testing and ideally POC diagnosis and treatment of syphilis, thereby mitigating transmission.

In 2011, it was estimated that $25 \%$ of individuals living with HIV in Canada were unaware of their HIV status and not connected to necessary treatment and counselling services [43]. Nearly one-quarter of participants reported no previous HIV testing. This study identified four new HIV cases by HIV POC testing; all had reported previously testing negative for HIV, which highlights the importance of regular testing of high risk populations. All four newly diagnosed cases were confirmed positive by standard testing, informed of their standard test result, and successfully referred to a HIV clinic for ongoing care and treatment. Overall, one in five male study participants reported sexual contact with the same sex and three of the four newly identified HIV cases were MSM, a population that represented $41 \%$ of all newly reported HIV cases among males in Alberta in 2011 [11].

Although, we purposely decided to offer testing at existing outreach sites that were accessed by high risk population represented in the existing outbreak, our study is limited by a nonrandom sample within these settings, which may have resulted in a selection bias and thus may limit the generalizability of our results. Our study participants had accessed Outreach sites and had consented to standard testing which may have inflated the proportion of persons accepting POC testing. However our data does agree with data from other researches at both incarceration sites and 
MSM sites [29, 30]. Additionally, the majority of those who participated had been tested in the past and the majority of those who tested positive for syphilis were previously treated. This made it difficult to evaluate the impact of introducing syphilis POC testing in these settings; however, we were able to focus on the feasibility of the strategy and the performance of the test in these outreach settings. In summary, our study showed that offering both HIV and syphilis POC testing to at risk populations in outreach settings in Edmonton, Canada was feasible as defined by the high proportion who completed POC testing. Additionally, the SD Bioline 3.0 Syphilis Test and INSTI HIV-1/HIV-2 Antibody test performed well in these settings when compared to standard testing. We found undiagnosed cases of syphilis and HIV and linked them to treatment and care. However, our ability to evaluate the impact and value of syphilis POC testing in these settings was limited by the small sample size, high prevalence of previously treated syphilis, and low prevalence of new cases of infectious syphilis. Future research should evaluate the usefulness of dual syphilis POC tests in similar settings. HIV POC testing may be helpful in reaching undiagnosed HIV infected, particularly when delivered to at risk populations such as MSM in social settings such as bathhouses.

\section{Conflict of Interests}

All authors declared that there is no conflict of interests.

\section{Acknowledgments}

The authors would like to thank the participants of the study; the Edmonton STI Clinic and Outreach Program (Barbara Anderson, Jody Brietzke, Patsy Conroy, Shyla Genoway, Sharyn Hewitt, Josettie Li, Penny Parker, Tracy Parnell, Kelly Rocco, and Julia Whitford); Catherine Litwin and Lisa Daye for assistance with data entry; the staff at the Alberta Provincial Laboratory for Public Health; and Dr. Rosanna Peeling for advice on selection of syphilis kit and assistance with kit procurement. This work was supported by grants from the Public Health Agency of Canada, Edmonton Inner City Health Research and Education Network, and by inkind support from Alberta Health Services. Preliminary data from this study were presented as a late breaker poster at the International Society for STD Research, Quebec City, Canada, July 10-13, 2011 J. Bergman; S. Plitt; J. Gratrix; P. Conroy; J. Li; K. Rocco; J. Fenton; C. Archibald; T. Wong; A. E. Singh. Preliminary Report on Experience with point-of-care Syphilis; and HIV Testing in Hard-to-Reach Populations in Outreach Settings in Edmonton, Canada (Sex Transm Infect 2011; 87; Suppl 1).

\section{References}

[1] D. Patrick, T. Wong, and R. Jordan, "Sexually transmitted infections in Canada: recent resurgence threatens national goals," Canadian Journal of Human Sexuality, vol. 6, pp. 149146, 2000.

[2] Public Health Agency of Canada, "Report on Sexually Transmitted Infections in Canada: 2008," 2010, http://www.phac-
aspc.gc.ca/std-mts/report/sti-its2008/PDF/10-047-STI_report_ eng-rl.pdf.

[3] Public Health Agency of Canada, "Syphilis. Canadian Guidelines on Sexually Transmitted Infections," January 2010, http:// www.phac-aspc.gc.ca/std-mts/sti-its/cgsti-ldcits/section-5-10eng.php.

[4] Government of Alberta, "Syphilis outbreak-cases continue to rise in Alberta," News Release March 2007, http://www .gov.ab.ca/acn/200703/2114837853E7C-B959-29A4-3A5E50AC A420B304.html.

[5] Government of Alberta, "The Syphilis Outbreak in Alberta. Office of the Chief Medical Officer of Health," December 2010, http://www.health.alberta.ca/documents/STI-Syphilis-Report2010.pdf.

[6] J. Gratix, L. Honish, L. Mashinter et al., "Case series descriptive analysis of a primary syphilis outbreak in Edmonton, Alberta, July 2004-April 2006," Canada Communicable Disease Report, vol. 33, no. 6, pp. 61-67, 2007.

[7] Unpublished data, Alberta Health Services, Edmonton, Alberta, Canada.

[8] Government of Alberta, "Notifiable Sexually Transmitted Infections 2011 Annual Report," http://www.health.alberta.ca/documents/STI-ND-Annual-Report-2011.pdf.

[9] J. Sexton, G. Garnett, and J.-A. Røttingen, "Metaanalysis and metaregression in interpreting study variability in the impact of sexually transmitted diseases on susceptibility to HIV infection," Sexually Transmitted Diseases, vol. 32, no. 6, pp. 351-357, 2005.

[10] J.-A. Røttingen, W. D. Cameron, and G. P. Garnett, "A systematic review of the epidemiologic interactions between classic sexually transmitted diseases and HIV: how much really is known?" Sexually Transmitted Diseases, vol. 28, no. 10, pp. 579-597, 2001.

[11] Government of Alberta, "HIV and AIDS in Alberta 2011 Annual Report," November 2012, http://www.health.alberta .ca/documents/STI-HIV-AIDS-Report-2011.pdf.

[12] A. Singh, S. Hill, and S. Shafran, "Non-return rates for HIV testing: results of a 3-month retrospective review at an STD clinic in 1999," Canada Communicable Disease Report, vol. 26, no. 18, pp. 152-155, 2000.

[13] S. Campbell and Y. Fedoriw, "HIV testing near the patient: changing the face of HIV testing," Clinics in Laboratory Medicine, vol. 29, no. 3, pp. 491-501, 2009.

[14] P. Ward, "Near-patient testing will improve the control of sexually transmitted infections: the arguments in favour," Sexually Transmitted Infections, vol. 82, no. 6, pp. 506-508, 2006.

[15] R. W. Peeling, D. Mabey, A. Herring, and E. W. Hook III, "Why do we need quality-assured diagnostic tests for sexually transmitted infections?" Nature Reviews Microbiology, vol. 4, no. 12, pp. 909-921, 2006.

[16] R. W. Peeling and H. Ye, "Diagnostic tools for preventing and managing maternal and congenital syphilis: an overview," Bulletin of the World Health Organization, vol. 82, no. 6, pp. 439446, 2004.

[17] D. C. Mabey, K. A. Sollis, H. A. Kelly et al., "Point-of-care tests to strengthen health systems and save newborn lives: the case of syphilis," PLOS Medicine, vol. 9, no. 6, Article ID e1001233, 2012.

[18] B. M. Branson, "State of the art for diagnosis of HIV infection," Clinical Infectious Diseases, vol. 45, no. 4, pp. S221-S225, 2007.

[19] J. H. Nichols, "Point of Care Testing," Clinics in Laboratory Medicine, vol. 27, no. 4, pp. 893-908, 2007. 
[20] L. Greer and G. D. Wendel Jr., "Rapid Diagnostic Methods in Sexually Transmitted Infections," Infectious Disease Clinics of North America, vol. 22, no. 4, pp. 601-617, 2008.

[21] J. D. Tucker, J. Bu, L. B. Brown, Y.-P. Yin, X.-S. Chen, and M. S. Cohen, "Accelerating worldwide syphilis screening through rapid testing: a systematic review," The Lancet Infectious Diseases, vol. 10, no. 6, pp. 381-386, 2010.

[22] D. Mabey, R. W. Peeling, R. Ballard et al., "Prospective, multicentre clinic-based evaluation of four rapid diagnostic tests for syphilis," Sexually Transmitted Infections, vol. 82, no. 5, pp. v13v16, 2006.

[23] B. E. Lee, S. Plitt, J. Fenton, J. K. Preiksaitis, and A. E. Singh, "Rapid HIV tests in acute care settings in an area of low HIV prevalence in Canada," Journal of Virological Methods, vol. 172, no. 1-2, pp. 66-71, 2011.

[24] Government of Alberta, "Alberta Treatment Guidelines for Sexually Transmitted Infections (STI) in Adolescents and Adults," 2012, http://www.health.alberta.ca/documents/STI-TreatmentGuidelines-2012.pdf.

[25] Standard Diagnostics, Inc., "SD Bioline Syphilis 3.0 Test Product Insert," March 2010, http://www.standardia.com/html_e/ mn03/mn03_01_00.asp?intId=28.

[26] Alberta Health Services-The Provincial Laboratory for Public Health, The Division of Medical MicrobiologyUniversity of Alberta, "Guide to Services," 2013, http:// www.provlab.ab.ca/guide-to-services.pdf.

[27] BioLytical Laboratories, "INSTI HIV-1/HIV-2 Antibody Test Product Insert,” 2007, http://www.biolytical.com/insti_doc/CEInsert.pdf.

[28] Alberta Health Services, "The Provincial Laboratory for Public Health. Laboratory Bulletin: HIV, HCV, and HBV serology testing change," June 2011, http://www.provlab.ab.ca/LabBulletin2011/HIV_HCV_HBV_Serology_TestingChange_Bulletin_June_9_2011.pdf.

[29] F. Spielberg, B. M. Branson, G. M. Goldbaum et al., "Choosing HIV counseling and testing strategies for outreach settings: a randomized trial," Journal of Acquired Immune Deficiency Syndromes, vol. 38, no. 3, pp. 348-355, 2005.

[30] C. G. Beckwith, S. Atunah-Jay, J. Cohen et al., "Feasibility and acceptability of rapid HIV testing in jail," AIDS Patient Care and STDs, vol. 21, no. 1, pp. 41-47, 2007.

[31] L. F. Haynes, J. E. Korte, B. E. Holmes et al., "HIV rapid testing in substance abuse treatment: implementation following a clinical trial," Evaluation and Program Planning, vol. 34, no. 4, pp. 399406, 2011.

[32] D. L. Pugatch, B. G. Levesque, M. A. Lally et al., "HIV testing among young adults and older adolescents in the setting of acute substance abuse treatment," Journal of Acquired Immune Deficiency Syndromes, vol. 27, no. 2, pp. 135-142, 2001.

[33] K. E. Bowles, H. A. Clark, E. Tai et al., "Implementing rapid HIV testing in outreach and community settings: results from an advancing HIV prevention demonstration project conducted in seven U.S. cities," Public Health Reports, vol. 123, no. 3, pp. 7885, 2008.

[34] M. Siedner, V. Zapitz, M. Ishida, R. De La Roca, and J. D. Klausner, "Performance of rapid syphilis tests in venous and fingerstick whole blood specimens," Sexually Transmitted Diseases, vol. 31, no. 9, pp. 557-560, 2004.

[35] M. Hernández-Trejo, B. Hernández-Prado, F. Uribe-Salas, L. Juárez-Figueroa, and C. J. Conde-González, "Maternal and congenital syphilis in two Mexican hospitals: evaluation of a rapid diagnostic test," Revista de Investigacion Clinica, vol. 58, no. 2, pp. 119-125, 2006.

[36] L. Juárez-Figueroa, F. Uribe-Salas, S. García-Cisneros, M. Olamendi-Portugal, and C. J. Conde-Glez, "Evaluation of a rapid strip and a particle agglutination tests for syphilis diagnosis," Diagnostic Microbiology and Infectious Disease, vol. 59, no. 2, pp. 123-126, 2007.

[37] A. R. Castro, J. Esfandiari, S. Kumar et al., "Novel point-ofcare test for simultaneous detection of nontreponemal and treponemal antibodies in patients with syphilis," Journal of Clinical Microbiology, vol. 48, no. 12, pp. 4615-4619, 2010.

[38] Y. Jafari, R. W. Peeling, S. Shivkumar, C. Claessens, L. Joseph, and N. P. Pai, "Are Treponema pallidum specific rapid and point-of-care tests for syphilis accurate enough for screening in resource limited settings? Evidence from a meta-analysis," PLoS ONE, vol. 8, no. 2, 2013.

[39] A. Herring, R. Ballard, D. Mabey, and R. W. Peeling, "Evaluation of rapid diagnostic tests: syphilis," Nature Reviews Microbiology, vol. 4, no. 12, pp. S33-S40, 2006.

[40] Y.-P. Yin, X.-C. Chen, W.-H. Wei et al., "A dual point-of-care test shows good performance in simultaneously detecting nontreponemal and treponemal antibodies in patients with syphilis: a multisite evaluation study in China," Clinical Infectious Diseases, vol. 56, pp. 659-665, 2013.

[41] World Health Organization, "The use of rapid syphilis tests," 2006, http://whqlibdoc.who.int/hq/2006/TDR_SDI_06.1_ eng.pdf.

[42] A. E. Singh, K. Sutherland, B. Lee, J. L. Robinson, and T. Wong, "Resurgence of early congenital syphilis in Alberta," CMAJ, vol. 177, no. 1, pp. 33-36, 2007.

[43] Public Health Agency of Canada, "Summary: Estimates of HIV Prevalence and Incidence in Canada," 2011, http:// www.phac-aspc.gc.ca/aids-sida/publication/survreport/assets/ pdf/estimat2011-eng.pdf. 


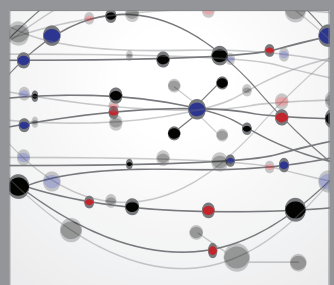

The Scientific World Journal
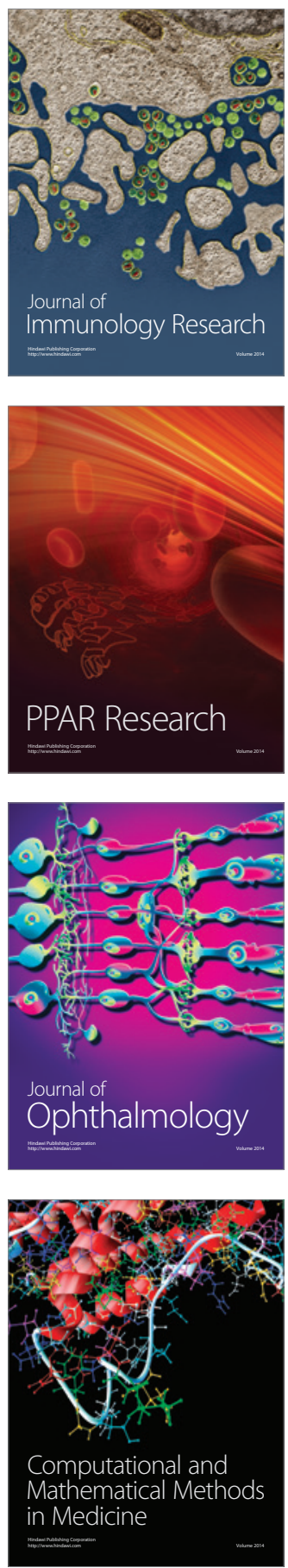

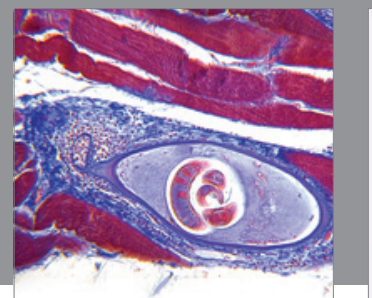

Gastroenterology

Research and Practice
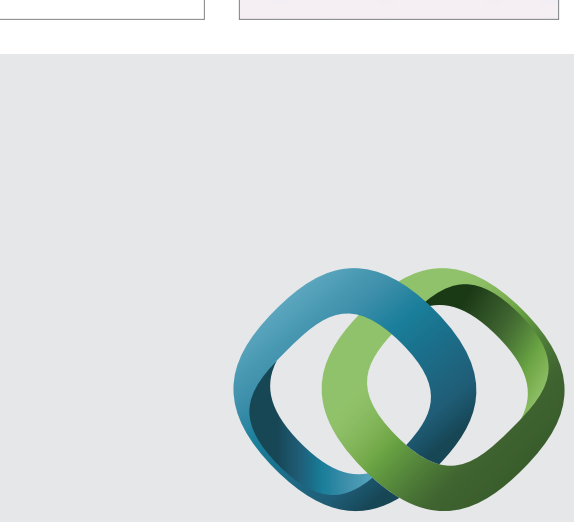

\section{Hindawi}

Submit your manuscripts at

http://www.hindawi.com
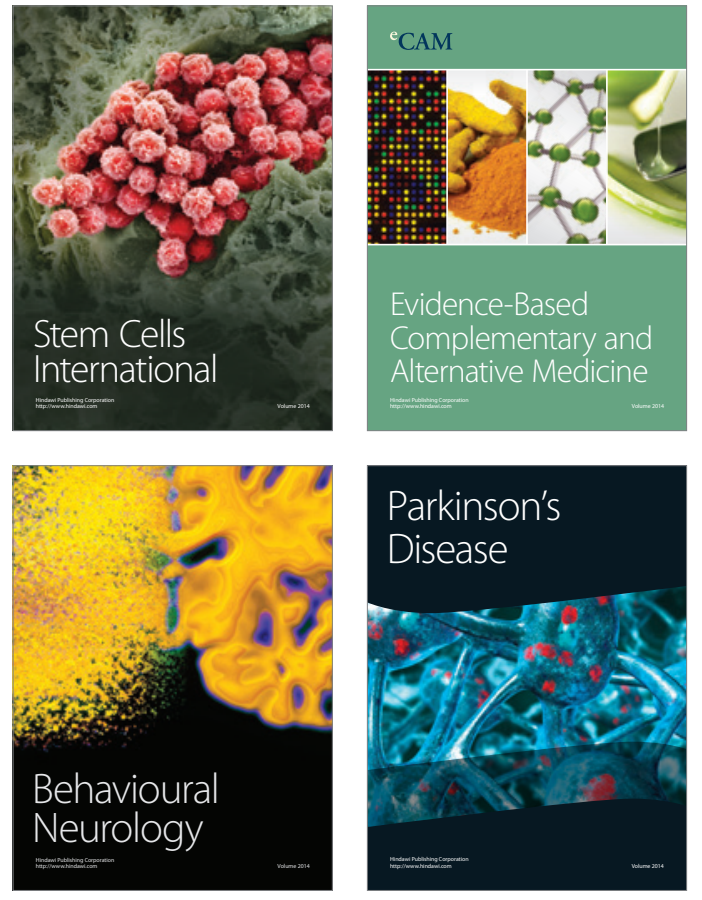
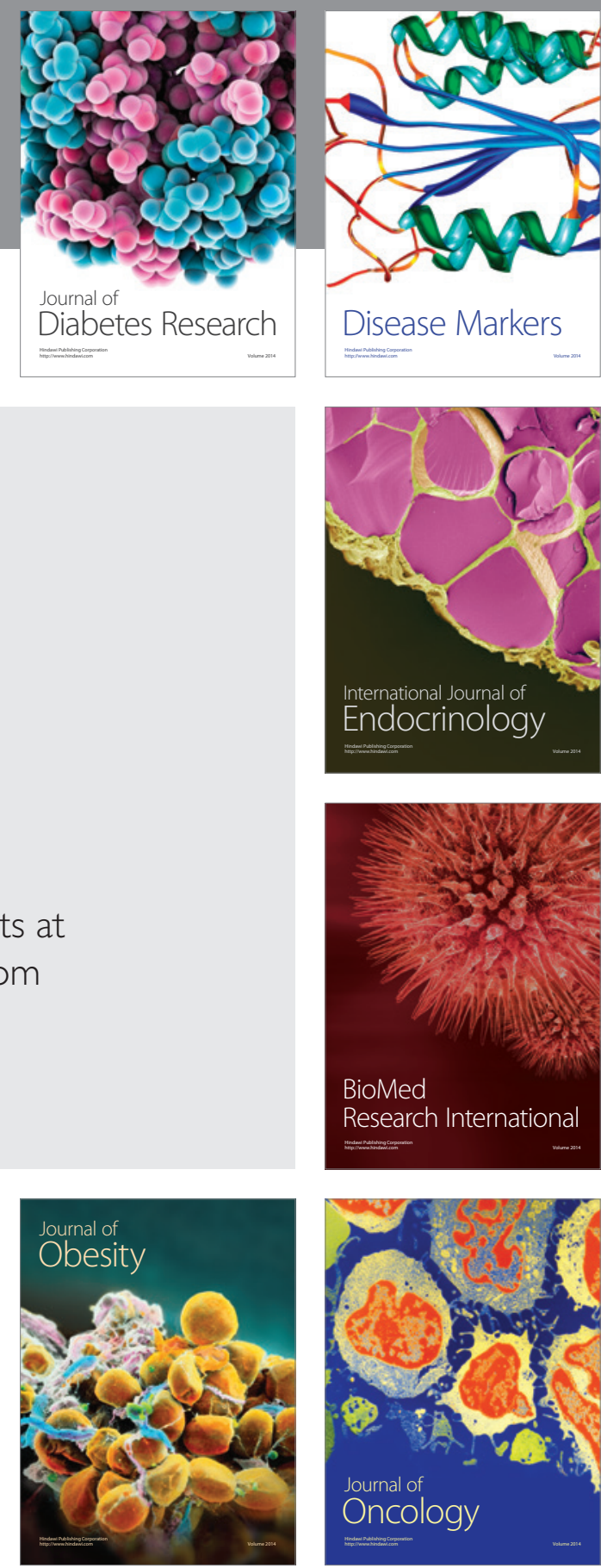

Disease Markers
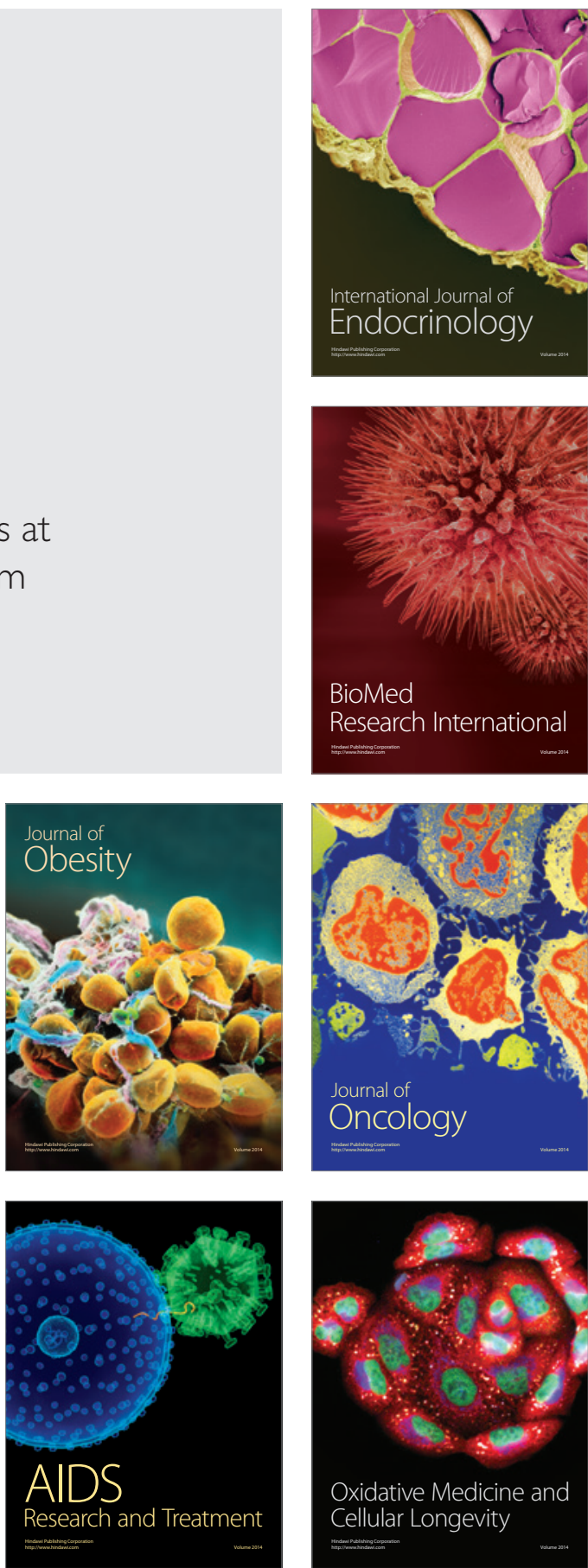